\title{
Gentamicin-collagen sponge reduces sternal wound complications after heart surgery: A controlled, prospectively randomized, double-blind study
}

\author{
Christoph Schimmer, MD, ${ }^{\mathrm{a}}$ Mehmet Özkur, MD, ${ }^{\mathrm{a}}$ Bhanu Sinha, PhD, ${ }^{\mathrm{b}}$ Johannes Hain, ${ }^{\mathrm{c}}$ Armin Gorski, MD, \\ Benjamin Hager, ${ }^{a}$ and Rainer Leyh, $\mathrm{MD}, \mathrm{PhD}^{\mathrm{a}}$
}

\begin{abstract}
Objective: Prophylactic retrosternal placement of a gentamicin-collagen sponge has been the subject of several recent clinical studies and is a matter of controversy. The present study is the first controlled, prospective, randomized, double-blind, single-center study to investigate the efficacy of a retrosternal gentamicin-collagen sponge in reducing sternal wound complications after heart surgery.
\end{abstract}

\begin{abstract}
Methods: From June 2009 to June 2010, 720 consecutive patients who underwent median sternotomy were assigned to a control placebo group (collagen sponge) or an intervention group (gentamicin-collagen sponge). All patients received guideline-compliant perioperative antibiotic prophylaxis. The primary end point was the occurrence of deep sternal wound infections within 30 days of index surgery (follow-up period). Secondary end points were the occurrence of superficial sternal wound infections requiring treatment, as well as further clinical parameters, including revision, bleeding volume, and need for transfusions during the follow-up period.
\end{abstract}

Results: A total of 720 of 994 patients $(72.4 \%$ ) were enrolled (control group: $n=367$ vs intervention group: $\mathrm{n}=353$ ). Risk factors for sternal wound infection and demographic variables were comparable in the 2 groups. The incidence of deep sternal wound infections was 13 of $367(3.52 \%)$ in the control group versus 2 of 353 $(0.56 \%)$ in the intervention group $(P=.014$; adjusted odds ratio, $0.15 ; 95 \%$ confidence interval, $0.02-0.69)$. The numbers needed to treat relation for all sternal wound infections and deep sternal wound infections were 26 and 33, respectively. No statistically significant differences were demonstrated concerning secondary end points, such as postoperative bleeding and transfusion of red cell units, thrombocytes, and fresh-frozen plasma.

Conclusions: Routine prophylactic retrosternal use of a gentamicin-collagen sponge in patients undergoing cardiac surgery significantly reduces deep sternal wound infections. (J Thorac Cardiovasc Surg 2012;143:194-200)

Median sternotomy is the method of first choice in most cardiac surgical interventions. Postoperative sternal wound infections are reported to occur with an incidence of approximately $0.5 \%$ to $8 \%{ }^{1}$ However, particularly in the case of deep sternal wound infections (DSWIs) they are associated with high mortality rates of $14 \%$ to $47 \% .^{2}$ The causes of DSWI are multifactorial. Risk factors include patient-related factors (endogenous) and those due to the surgical intervention and hospital stay (exogenous). A number of prospective and retrospective studies have underscored the significance of risk factors,

From the Department of Cardiothoracic and Thoracic Vascular Surgery, ${ }^{a}$ University Hospital Würzburg, Germany; and Institute of Hygiene and Microbiology, ${ }^{\mathrm{b}}$ and Chair of Mathematics VIII (Statistics), ${ }^{\mathrm{c}}$ Institute of Medicine, University of Würzburg, Germany.

This study was supported by RESORBA Wundversorgung GmbH \& Co KG, 90475 Nürnberg. All sponges used in this study were provided free of charge.

Disclosures: Authors have nothing to disclose with regard to commercial support.

Received for publication April 4, 2011; revisions received May 10, 2011; accepted for publication May 25, 2011; available ahead of print Sept 1, 2011.

Address for reprints: Christoph Schimmer, MD, Department for Thoracic, Cardiac and Thoracic Vascular Surgery, University Hospital of Würzburg, Oberdürrbacherstraße 6, 97080 Würzburg, Germany (E-mail: Schimmer_c@klinik.uni-wuerzburg.de).

$0022-5223 / \$ 36.00$

Copyright (c) 2012 by The American Association for Thoracic Surgery doi:10.1016/j.jtcvs.2011.05.035 because they are targets for preventive measures. ${ }^{2-8}$ The basic requirements for uncomplicated wound healing are aseptic surgery, perfect osteosynthesis, and optimal antimicrobial prophylaxis. Local antibiotic-eluting products are already being used in the treatment of osteomyelitis, soft-tissue infections, and mediastinitis. ${ }^{9-12}$ The effectiveness of the routine retrosternal placement of a gentamicin-collagen sponge before suture closure is currently a matter of some controversy. ${ }^{13-16}$ Local use results in initially high local concentrations at the implantation site without causing toxic serum levels. This counters the risk of resistance development while at the same time avoiding systemic adverse effects of the aminoglycoside, for example, ototoxicity and nephrotoxicity. ${ }^{9,12,17-19}$ However, the carrier is a foreign body that should be removed promptly once the active substance has been largely eluted or made of fully biodegradable material to prevent persistence of an infective focus. Thus, a collagen matrix seems to be suitable in situations in which removal is not a viable option, such as sternotomy. The present study is the first controlled, prospectively randomized, double-blind, single-center study to investigate the efficacy of a gentamicin-collagen sponge in reducing sternal wound 


\section{Abbreviations and Acronyms}

DSWI $=$ deep sternal wound infection

SSWI $=$ superficial sternal wound infection

complications after heart surgery and meets the criteria of evidence grade $1 b$.

\section{MATERIALS AND METHODS}

In the period between June 15, 2009, and June 30, 2010, 994 operations were performed on the heart and thoracic aorta via median sternotomy at the Clinic for Thoracic, Cardiac and Thoracic Vascular Surgery of Würzburg University Hospital. A total of 800 of 994 patients $(80.5 \%)$ met the inclusion criteria, which were defined as follows: male and female legally competent patients, elective and emergency, age 18 years or older, cardiac surgery undergoing first or resternotomy, ability to provide written informed consent, and no preoperative signs of thoracic inflammation. Exclusion criteria were defined as follows: existing osteitis, immunosuppressive therapy, known hypersensitivity to aminoglycoside antibiotics, concurrent immunologic disease, pregnancy or lactation, participation in another clinical study, inability to give consent to participate in the study, and refusal to participate in the study before or during the follow-up period (Figure 1).

For the study, 400 pure collagen sponges $\left(20 \times 5 \times 0.5 \mathrm{~cm} ; 1 \mathrm{~cm}^{2}\right.$ containing $2.8 \mathrm{mg}$ native collagen fibrils of equine origin; placebo) and 400 identical gentamicin-collagen sponges (also containing $2 \mathrm{mg}$ gentamicin sulphate, equivalent to $1.10-1.43 \mathrm{mg}$ gentamicin; verum) were used. The 2 sponges looked identical and could not be differentiated by the surgeon on the basis of their appearance or haptic characteristics. The study sponges (verum + placebo) were provided free of charge by RESORBA Wundversorgung GmbH \& Co KG, 90475 Nürnberg. An agreement on the cooperation was drawn up with the Legal Department of Würzburg University Hospital. Financial remuneration and the possibility of influencing the publication of the findings were ruled out by the agreement. None of the parties involved received any remuneration from RESORBA Wundversorgung $\mathrm{GmbH} \& \mathrm{Co} \mathrm{KG}$ before, at the time of the study, or at the time of publication of the findings. The corresponding author had full access to all data and final responsibility for the decision to submit for publication. All criteria

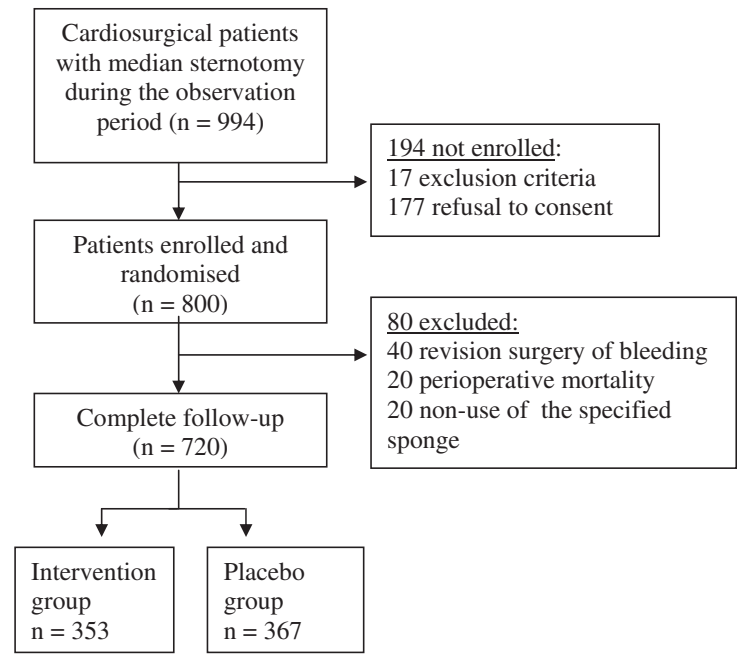

FIGURE 1. Trial profile. demanded of a randomized, double-blind study were met at all times, so that the study meets the criteria of the Oxford Centre for Evidence-based Medicine for evidence grade 1b. Randomization and blinding were carried out by the Centre for Clinical Studies of Würzburg University Hospital. After randomization, the 400 gentamicin-collagen sponges and the 400 placebo sponges were labeled with a sticker showing the randomization number. Once all the sponges were used, the data were unblinded and analyzed by the Institute of Mathematics and Informatics, Chair for Statistics of Würzburg University. The study was approved by the Ethics Committee of Würzburg University Hospital (reference No. 69/09). This trial is registered at Current Controlled Trials Limited, London with the assign ISRCTN 20849.

\section{Primary and Secondary End Points}

All patients were examined once daily up to the time of discharge for wound healing and signs of infection. Preoperatively and on postoperative days 1, 2, 4, and 7 and on the day before discharge, the leucocyte count, C-reactive protein, and thrombocyte count were determined. If infection was suspected, these parameters were also measured on an ad hoc basis. To detect further infections after discharge, patients were instructed to report any signs of infection and they were contacted by phone 30 days postoperatively. The primary end point of the study was the occurrence of a DSWI/mediastinitis within the 30-day follow-up period after index surgery. Secondary end points were the occurrence of a superficial sternal wound infection (SSWI) requiring treatment and the following parameters: revision, total bleeding volume, and need for transfusion during the followup period. DSWI and SSWI were defined on the basis of the criteria of the US Centers for Disease Control and Prevention as a positive bacterial culture from mediastinal tissue or fluid, and an intraoperative clinical picture of mediastinitis in the presence of one of the following: chest pain, sternal instability, fever greater than $38^{\circ} \mathrm{C}$, purulent secretion from the mediastinum, or positive bacterial culture from the blood or mediastinal drainage fluid. An SSWI was deemed present if there was microbiological evidence of microbes in the cutaneous or subcutaneous tissue at the site of the incision, the incision was purposely reopened, or at least one of the following secondary diagnoses was made: purulent secretion from the superficial incision with or without laboratory confirmation, positive bacterial culture from a fluid or tissue sample taken from the superficial incision under aseptic conditions, and at least 1 sign/symptom of infection: redness, heat, pain or swelling. ${ }^{20}$

\section{Surgical Procedure}

After complete adaptation of the pericardium and preliminary placement of the sternal wiring, the study sponge was implanted retrosternally without premoistening (Figure 2).

Sternal wiring was then performed. The closure techniques used were trans-/peristernal, figure of 8 , or band cerclages. The wound was then closed in layers by sutures and covered with a sterile dressing. Each patient routinely received guideline-compliant perioperative prophylaxis with intravenous cefuroxime ( $1.5 \mathrm{~g}$ twice per day) starting 30 minutes before the operation for up to 48 hours postoperatively. The preoperative, intraoperative, and postoperative protocol for preventing wound infections was not changed during the course of the study. There were no changes in the hygiene or surgical procedure during that time.

\section{Statistical Analysis}

Statistical analysis was performed by an independent statistician at the Institute of Mathematics and Informatics, Chair of Mathematics VIII (Statistics), University of Würzburg. The open source software R (version 2.12.1) was used. For ratio-scaled variables, a descriptive overview of the 2 groups (active drug vs placebo) was always prepared. The 2 groups were then analyzed with the Mann-Whitney $U$ test for significant 

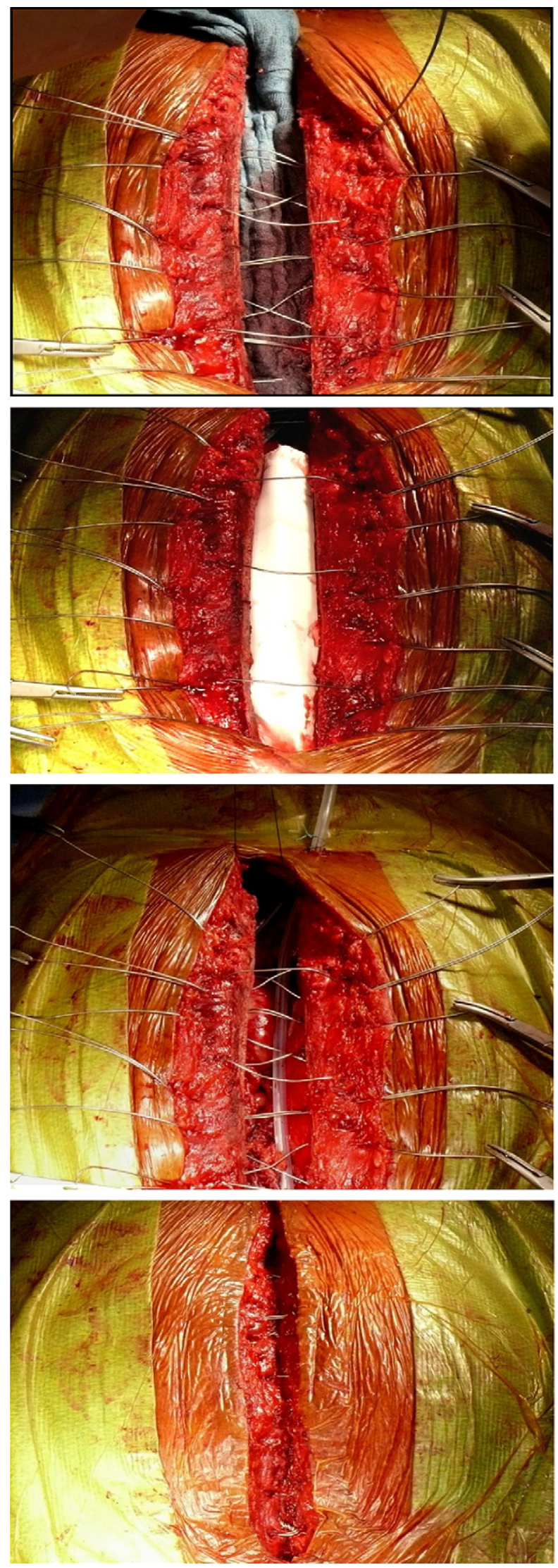

differences. A group overview was also prepared for nominal-scaled variables. To determine differences in these values, the chi-square test of independence was performed. If the variable was binomial (eg, gender), the Fisher exact test was applied and the odds ratio with a $95 \%$ confidence interval was calculated. The subgroup analysis was performed with the Fisher exact test or Mann-Whitney $U$ test according to the scale type of the corresponding measurement. For all tests in the subgroup analysis, the Benjamini-Hochberg correction of the significance level was conducted. Kaplan-Meier analysis was performed to estimate the mean time until the appearance of an infection. To compare the 2 estimates of the 2 groups, a log-rank test was done. The data were processed and analyzed while preserving the patient's anonymity.

\section{RESULTS}

A total of 720 of $800(90 \%)$ of the screened patients were included in the study analysis after the end of the 30-day follow-up period after index surgery. Some 80 of 800 patients $(10 \%)$ could not be evaluated, the reasons being revision surgery because of bleeding (40x), perioperative mortality not associated with a wound infection (20x), and non-use of the specified sponge (20x). Some 353 of 720 patients $(49 \%)$ were assigned to group I (intervention group; active drug), and 367 of 720 patients (51.0\%) were assigned to group II (control group; placebo). After the 2 groups were unblinded, it was shown that the intervention and placebo groups differed statistically in a few points only. There were no significant differences with regard to the most frequently mentioned risk factors (obesity, diabetes, chronic obstructive pulmonary disease, age, $\mathrm{HbA}_{1 \mathrm{c}}$, renal impairment, and peripheral arterial obstructive disease). There were differences in preoperative factors (eg, gender) $(P=.041)$. In the intervention group, $70.5 \%$ of the patients were men versus $77.4 \%$ in the placebo group. The difference in proportions was statistically significant $(P=.041)$. In addition, an ejection fraction of less than $30 \%$ was found in $4.8 \%$ of the patients in the intervention group and in $9.0 \%$ of the patients in the placebo group. The difference of $4.1 \%$ was statistically significant $(P=.029)$ (Table 1).

The incidence of SSWI and DSWI was 11 of 367 patients $(3 \%)$ and 13 of 367 patients $(3.52 \%)$, respectively, in the control group versus 7 of 353 patients $(1.98 \%)$ and 2 of 353 patients $(0.56 \%)$, respectively, in the intervention group (Table 2).

The probability of developing a DSWI was lower in the intervention group $(66.3 \%)$ than in the placebo group. The non-infection probability in the follow-up period was significantly higher in the verum group than in the placebo group ( $\log$-rank $P=.00529)$. The numbers needed to treat relation for all sternal wound infections and DSWI were $1: 26$ and 1:33, respectively (Figure 3 ).

FIGURE 2. Gentamicin sponge placement and sternal closure. Placement of the sponge before sternal closure. 
TABLE 1. Patient characteristics, comorbidities, and procedure-related variables

\begin{tabular}{|c|c|c|c|c|}
\hline & Active drug & Placebo & Total & $P$ value \\
\hline \multicolumn{5}{|l|}{ Preoperative } \\
\hline $\operatorname{Men}(\%)$ & $249(70.5)$ & $284(77.4)$ & $533(74.0)$ & .041 \\
\hline Age, y & $69(33-85)$ & $69(29-87)$ & $69(29-87)$ & .800 \\
\hline BMI & $28.1 \pm 4.5$ & $28.1 \pm 4.3$ & $28.1 \pm 4.4$ & .763 \\
\hline COPD $(\%)$ & $50(14.2)$ & $49(13.4)$ & $99(13.8)$ & .829 \\
\hline Diabetes mellitus & $99(28.0)$ & $119(32.4)$ & $218(30.3)$ & .224 \\
\hline $\mathrm{HbA}_{1 \mathrm{c}}(\%)$ & $6.25 \pm 0.93$ & $6.30 \pm 0.96$ & $6.27 \pm 0.95$ & .387 \\
\hline Consuming diseases & $11(3.1)$ & $5(1.4)$ & $16(2.2)$ & .133 \\
\hline Myocardial infarction (\%) & $15(4.2)$ & $12(3.3)$ & $27(3.8)$ & .558 \\
\hline $\mathrm{EF}<30 \%$ & $17(4.8)$ & $33(9.0)$ & $50(6.9)$ & .029 \\
\hline Cardiogenic shock $(\%)$ & $8(2.3)$ & $5(1.4)$ & $13(1.8)$ & .412 \\
\hline Renal impairment (\%) & $79(22.4)$ & $91(24.8)$ & $170(23.6)$ & .438 \\
\hline Creatinine (mg/dL) & $1.06 \pm 0.73$ & $1.10 \pm 0.60$ & $1.08 \pm 0.67$ & .010 \\
\hline Urea (mg/dL) & $40.44 \pm 18.95$ & $41.71 \pm 22.30$ & $41.09 \pm 20.72$ & .676 \\
\hline GFR & $79.6 \pm 25.1$ & $76.8 \pm 25.1$ & $78.2 \pm 25.1$ & .088 \\
\hline PAOD $(\%)$ & $41(11.6)$ & $35(9.5)$ & $76(10.6)$ & .397 \\
\hline Osteoporosis (\%) & $7(2.0)$ & $5(1.4)$ & $12(1.7)$ & .571 \\
\hline Hyperthyroidism (\%) & $8(2.3)$ & $5(1.4)$ & $13(1.8)$ & .412 \\
\hline Hypothyroidism (\%) & $22(6.2)$ & $22(6.0)$ & $44(6.1)$ & 1.000 \\
\hline Leucocyte count $(\mathrm{n} * 1000 / \mu \mathrm{L})$ & $7.94 \pm 3.61$ & $7.54 \pm 2.79$ & $7.74 \pm 3.22$ & 0.112 \\
\hline CRP (mg/dL) & $1.18 \pm 2.87$ & $1.20 \pm 2.43$ & $1.19 \pm 2.66$ & 0.200 \\
\hline Reoperation & $8(2.3)$ & $25(6.8)$ & $33(4.6)$ & .004 \\
\hline Emergency & $71(20.1)$ & $63(17.2)$ & $134(18.6)$ & .339 \\
\hline \multicolumn{5}{|l|}{ Intraoperative } \\
\hline Type of procedure & & & & .197 \\
\hline CABG (+HLM) & $186(52.7)$ & $195(53.1)$ & $381(52.9)$ & \\
\hline OPCAB & $30(8.5)$ & $29(7.9)$ & $59(8.2)$ & \\
\hline Isolated valve surgery & $63(17.8)$ & $86(23.4)$ & 149 (20.7) & \\
\hline Combination intervention & $72(20.4)$ & $55(14.7)$ & $126(17.5)$ & \\
\hline Other & $2(0.6)$ & $3(0.8)$ & $5(0.7)$ & \\
\hline \multicolumn{5}{|l|}{ Procedure-related variables } \\
\hline Operating time, $\min$ & $252.5 \pm 60.0$ & $251.4 \pm 59.9$ & $252.0 \pm 59.9$ & .849 \\
\hline Bypass time, min & $123.0 \pm 45.4$ & $120.4 \pm 42.3$ & $121.6 \pm 43.8$ & .455 \\
\hline Clamping time, $\min$ & $87.9 \pm 38.3$ & $87.2 \pm 35.4$ & $87.5 \pm 36.8$ & .622 \\
\hline \multicolumn{5}{|l|}{ Sternal closure } \\
\hline$\geq 4$ figures of 8 & $183(51.8)$ & $209(56.9)$ & $392(54.4)$ & \\
\hline$<4$ figures of 8 & $4(1.1)$ & $2(0.5)$ & $6(0.8)$ & \\
\hline$\geq 7$ conventional & $135(38.2)$ & $127(34.6)$ & $262(36.4)$ & \\
\hline$<7$ conventional & $14(4.0)$ & $11(3.0)$ & $25(3.5)$ & \\
\hline Other & $18(4.8)$ & $18(4.9)$ & $35(4.9)$ & \\
\hline \multicolumn{5}{|l|}{ Postoperative } \\
\hline Ventilation time, $\min$ & 580.0 & 565.0 & 566.5 & .377 \\
\hline Transitory psychotic syndrome & $75(21.2)$ & $75(20.4)$ & $150(20.8)$ & .854 \\
\hline Thoracic vest & 27 (7.6) & $19(5.2)$ & $46(6.4)$ & .222 \\
\hline Tracheotomy & $9(2.5)$ & $8(2.2)$ & $17(2.4)$ & .809 \\
\hline
\end{tabular}

Boldface indicates significant parameters. BMI, Body mass index; COPD, chronic obstructive pulmonary disease; $E F$, ejection fraction; GFR, glomerular filtration rate; $P A O D$, peripheral arterial obstructive disease; $C R P$, C-reactive protein; $C A B G$, coronary artery bypass grafting; $H L M$, heart-lung machine; $O P C A B$, off-pump coronary artery bypass.

Detected bacteria in the 15 patients with DSWI were coagulase-negative staphylococci $(68.4 \%)$, gram-negative bacteria (10.5\%), Propionibacterium acnes $(10.5 \%)$, and Staphylococcus aureus (5.3\%). With only 2 cases of DSWI in the gentamicin group, a difference between the 2 groups could not be detected.

\section{DISCUSSION}

The most serious form of postoperative sternal wound complication is DSWI. As a disease entity, DSWI occurs with an incidence of up to $8 \%{ }^{1}$ and is associated with high morbidity and mortality rates of $14 \%$ to $47 \%{ }^{2}$. This means that in Germany approximately 2000 patients 
TABLE 2. Primary and secondary end points

\begin{tabular}{|c|c|c|c|c|c|}
\hline & Active drug & Placebo & Total & $\boldsymbol{P}$ & OR $(95 \%$ CI $)$ \\
\hline \multicolumn{6}{|l|}{ Primary end points } \\
\hline All wound complications & $9(2.54)$ & $24(6.50)$ & $33(4.56)$ & .012 & $0.37(0.15-0.54)$ \\
\hline SSWI & $7(1.98)$ & $11(2.98)$ & $18(2.49)$ & .470 & $0.63(0.20-1.81)$ \\
\hline \multirow[t]{2}{*}{ DSWI } & $2(0.56)$ & $13(3.52)$ & $15(2.07)$ & .014 & $0.15(0.02-0.69)$ \\
\hline & Active drug & & Placebo & Total & $P$ \\
\hline \multicolumn{6}{|l|}{ Secondary end points } \\
\hline Revision & $17(4.8)$ & & $11(3.0)$ & $28(3.9)$ & .249 \\
\hline Bleeding volume, $\mathrm{mL}$ & $1411.63 \pm 1086.6$ & & $1335.7 \pm 991.0$ & $1372.9 \pm 1038.9$ & .650 \\
\hline Units of packed red cells & $2.5 \pm 2.7$ & & $2.2 \pm 2.8$ & $2.4 \pm 2.8$ & .103 \\
\hline Units of platelet concentrate & $0.7 \pm 1.2$ & & $0.8 \pm 1.5$ & $0.8 \pm 1.4$ & .275 \\
\hline Units of fresh-frozen plasma & $1.7 \pm 3.6$ & & $1.5 \pm 2.9$ & $1.6 \pm 3.3$ & .840 \\
\hline
\end{tabular}

annually develop this serious and potentially lifethreatening complication. Reducing sternal wound infections lowers mortality and morbidity. It also cuts costs, because it helps to avoid further surgical and pharmacologic therapies and shortens hospital stays. ${ }^{21-23}$ Economic aspects of mediastinitis were calculated in a matched case-control study by Graf and colleagues. ${ }^{21}$ They calculated that median overall costs of a coronary artery bypass grafting case were EUR 36,261 per infected patient compared with EUR 13,356 per control patient without infection $(P<.0001)$, implying additional costs of approximately EUR 23.000 per infection. A total of EUR 9154 after reimbursement was lost for every case of DSWI during the study period. Infection control measures for reducing DSWI therefore seem justified and will become cost-effective. ${ }^{21}$

The literature contains many articles on the efficacy of the prophylactic use of a gentamicin-impregnated fleece in surgery. ${ }^{9,10,12-16}$ Specifically, the literature contains 3

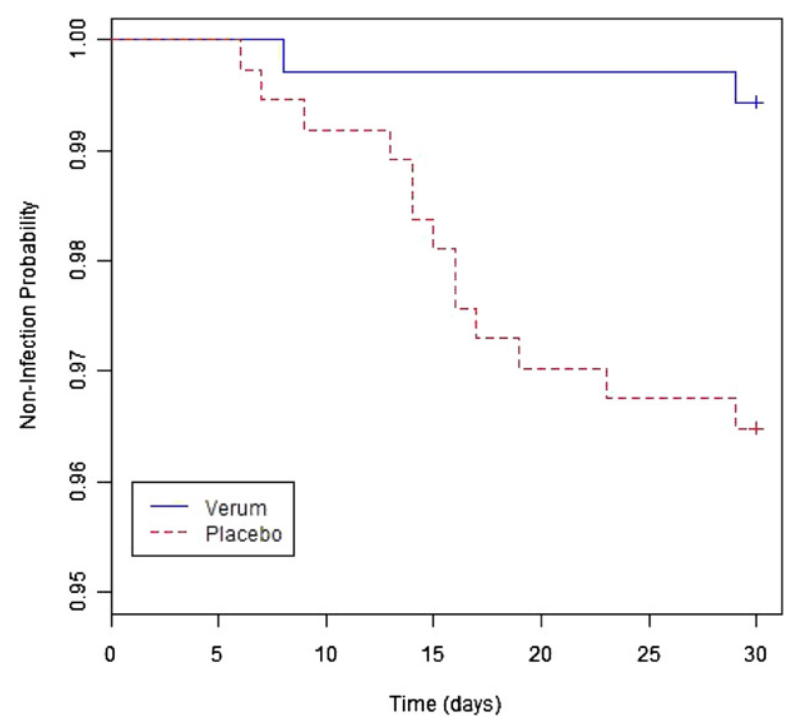

FIGURE 3. Kaplan-Meier plot. studies on patients undergoing cardiac surgery and the prophylactic use of a gentamicin sponge: The first study by Friberg and colleagues ${ }^{14}$ included 2000 patients exclusively undergoing coronary artery bypass grafting (LOGIP trial). A total of 967 patients (control group) received routine intravenous prophylaxis (isoxazolyl penicillin for 48 hours postoperatively) versus 398 patients (intervention group) who also had 2 gentamicin-collagen sponges (260 mg) implanted retrosternally (LOGIP trial). The rate of sternal wound complications was $4.3 \%$ in the intervention group versus $9.0 \%$ in the control group $(P<.001)$. This shows a significant beneficial effect of the additional use of a gentamicin sponge.

The results of the present study support the conclusion of Friberg and colleagues. ${ }^{14}$ In terms of gender distribution and age, the 2 study populations differed only marginally. However, with a mean body mass index of $28.1 \pm 4.4$ $\mathrm{kg} / \mathrm{m}^{2}$ (LOGIP, $26.4 \mathrm{~kg} / \mathrm{m}^{2}$ ), a diabetic proportion of $30.2 \%$ (LOGIP, 18.2), and a chronic obstructive pulmonary disease proportion of $13.7 \%$ (LOGIP, $5.6 \%$ ), the patients in our study had a higher overall risk for sternal wound infection. An important difference between the 2 studies concerns the technique of implanting the sponge. In the LOGIP study, Friberg and colleagues ${ }^{14}$ used 2 collagen-gentamicin sponges (260 mg gentamicin), whereas in our study only 1 sponge was implanted, equivalent to $130 \mathrm{mg}$ gentamicin, in the intervention group. Friberg and colleagues ${ }^{14}$ placed the 2 sponges between the 2 halves of the sternum, whereas in this study the sponges were positioned retrosternally. Which technique is superior and the optimum number of sponges to be used should be clarified in further studies. Retrosternal implantation of the sponge in the present study may have resulted in higher retrosternal gentamicin levels and consequently lower presternal levels. This could explain the nonsignificant reduction in SSWIs with a simultaneous significant reduction in mediastinitis. Friberg and colleagues ${ }^{14}$ observed that the group provided with a gentamicin sponge had a significantly higher rate of reoperations 
due to bleeding ( $4.0 \%$ vs $2.3 \%)$. Because of our study design with a placebo group, we are unable to draw any conclusions regarding a putatively higher reoperation rate, although our reoperation rate was comparable at $4.6 \%$. However, we found no statistical association with the development of sternal wound complications. Finally, it should be noted that the study subjects were not blinded in the study by Friberg and colleagues, ${ }^{14}$ which must be seen as a major limitation.

In the present study, the follow-up contact occurred 1 month after the index operation. There are reports of sternal wound complications up to 1 year, but more than $90 \%$ of these cases present within 1 month. This is also supported by the fact that throughout the study period no readmission for sternal wound infection occurred after 1 month. The design of the present study meets the criteria of evidencebased medicine of the highest quality for an individual study. This is a rarity in surgical research, because such a study design is usually difficult to realize in the surgical field. Both study groups showed no significant difference in the major risk factors. In some points (gender, ejection fraction $<30 \%$, and reoperation), both groups differed. An increased risk for a sternal wound infection was not found in any of the patients in the subgroup analyses in the present study. Concluding comparability of both groups is given.

The second study published on this topic was carried out by Eklund and colleagues ${ }^{13}$ in 542 patients. They demonstrated a reduction (without a significant influence) of SSWI and DSWIs brought about by the use of a gentamicin-releasing sponge $(4.0 \%$ vs $5.9 \%$ and $1.1 \%$ vs $1.9 \%$, respectively). This unblinded study was also performed exclusively with 542 patients undergoing elective coronary surgery, so that neither the statistical power nor the real picture of daily practice was represented.

The third and most recent study on this topic was published by Bennett-Guerrero and colleagues ${ }^{16}$ in 2010 . The study design chosen was similar to that of the LOGIP study. However, Bennett-Guerrero and colleagues ${ }^{16}$ were unable to confirm the results, and, on the basis of their data, they concluded that the collagen-gentamicin sponge is ineffective in preventing sternal wound infections in the context of cardiac surgery. The study included 1502 patients at 48 US hospitals. The inclusion and exclusion criteria of the study by Bennett-Guerrero and colleagues ${ }^{16}$ differed in several respects from both the present study and the LOGIP study. Unlike in the latter 2 studies, the study by BennettGuerrero and colleagues ${ }^{16}$ enrolled only patients with elective, non-emergency bypass or valve operations, and these patients were at increased risk for sternal wound infections. This higher risk was defined on the basis of diabetes or a body mass index of more than $30 \mathrm{~kg} / \mathrm{m}^{2}$. In our study, $18.6 \%$ of the patients underwent urgent or emergency surgery. In the study by Friberg and colleagues, ${ }^{14}$ this ratio was approximately $2.3 \%$. In addition, Bennett-Guerrero and colleagues ${ }^{16}$ excluded patients with an intraaortic balloon pump for shock or low-output syndrome. The proportion of these patients was not recorded in our study, but $1.8 \%$ of the patients had a cardiogenic shock. Other reasons for exclusion were a serum creatinine level in excess of $3 \mathrm{mg} / \mathrm{dL}$, the need for dialysis because of renal failure, significant alcohol abuse, or systemic immunosuppression at the time of the study. In our study, these parameters were not regarded as exclusion criteria. The patients in our study and the LOGIP study by Friberg and colleagues ${ }^{14}$ were exposed to a broader range of risk factors than the patients in the study by Bennett-Guerrero and colleagues. ${ }^{16}$ However, the overall risk of wound infection was higher in the US study ${ }^{16}$ than in our study and the LOGIP study ${ }^{14}$ because of the more stringent selection. As did Friberg and colleagues, ${ }^{14}$ Bennett-Guerrero and colleagues ${ }^{16}$ used 2 collagen-gentamicin sponges, each containing $130 \mathrm{mg}$ gentamicin, which were placed between the 2 sternal halves. As in the LOGIP study, a placebo sponge was not used, in contrast with the present study. Another difference among the studies concerns the number of participating hospitals. The present study was a single-center study, whereas the LOGIP study by Friberg and colleagues ${ }^{14}$ included 2 Swedish hospitals and the US study by Bennett-Guerrero and colleagues ${ }^{16}$ included 48 hospitals in total. It is assumed that a multicenter study is unsuitable for addressing the question being investigated. The aim is to evaluate the efficacy of a single procedure (ie, the use of a gentamicin-collage sponge) with respect to a complication with a low incidence and multifactorial genesis. This requires that one needs to keep all potential influencing factors as constant as possible. Otherwise, the positive effect of a procedure could be swamped by the overall influence of a wide range of factors. In the study by Friberg and colleagues, ${ }^{14}$ the results were compromised by the use of different sternal wiring methods. We now know that the patient presents with a broad range of risk factors a priori, but the surgical technique, the type of skin disinfection, the use of 2 pairs of gloves, the number of people present in the operating room, and many other factors also have an influence on wound infections. Thus, it can be speculated that despite all its positive aspects, a multicenter design is not suitable for addressing the question concerned in this case.

The US study by Bennett-Guerrero and colleagues ${ }^{16}$ also clearly showed how greatly surgical procedures differ. In the gentamicin group, for example, the patient's hair was not removed in the operating room in $22.6 \%$ of the cases, and nasal mupirocin was used preoperatively in $48.2 \%$ of the patients. Skin disinfection was not standardized $(57.5 \%$ povidone-iodine, $52.6 \%$ alcohol, $47.5 \%$ chlorhexidine-based), and various antibiotics were also administered preoperatively $(53.5 \%$ cefazolin, $23.4 \%$ cefuroxime, $1.9 \%$ ciprofloxacin, $30.5 \%$ vancomycin). It can 
therefore be speculated that, as in the study by Friberg and colleagues, ${ }^{14}$ these different variables may have led to a distortion of the results. Bennett-Guerrero and colleagues ${ }^{16}$ suspect that dissimilar microbial spectra may explain the differences in results. In our study, coagulase-negative staphylococci were the most common pathogens at $68.4 \%$ in the verum group and $50 \%$ in the placebo group. With only 2 cases of DSWI in the gentamicin group, a difference between the 2 groups could not be detected. This microbial spectrum is similar to that in the Swedish study by Friberg and colleagues, ${ }^{14}$ in which coagulase-negative staphylococci formed the largest group of pathogens. The US study ${ }^{16}$ showed a heterogeneous microbial spectrum. A significant difference relates to the number of $S$. aureus cases, which accounted for 2.4\% (1 case) in the present study, $21.7 \%$ in the Swedish study, ${ }^{14}$ and $37.3 \%$ in the US study. ${ }^{16}$ In our study and the Swedish study, ${ }^{14}$ no patients had methicillin-resistant $S$ aureus. By contrast, methicillin-resistant $S$ aureus infection was identified in $10.2 \%$ of the microbial isolates $(27.3 \%$ of the $S$ aureus cases) in the US study. ${ }^{16}$ The extent to which the dissimilar microbial spectra and possible antibiotic resistance influence the results cannot be clarified.

\section{CONCLUSIONS}

On the basis of the literature and the results obtained in this controlled, prospectively randomized, double-blind study, routine prophylactic retrosternal implantation of an antibiotic-releasing sponge has a beneficial effect (reduction in the incidence of sternal wound infections). Because mediastinitis is a multifactorial complication, an enormous risk reduction can never be achieved by implementing a single measure. Many individual measures must be applied that in the end will lead to a marked risk reduction. Nevertheless, each building block needs to be evaluated for efficacy.

\section{References}

1. Franco S, Herra AM, Atehortua M, Velez L, Botero J, Jaramillo J, et al. Use of steel bands in sternotomy closure: implications in high-risk cardiac surgical population. Interact Cardiovasc Thorac Surg. 2009;8:200-5.

2. El Oakley RM, Wright JE. Postoperative mediastinitis: classification and management. Ann Thorac Surg. 1996;61:1030-6.

3. Robicsek F, Fokin A, Cook J, Bhatia D. Sternal instability after midline sternotomy. J Thorac Cardiovasc Surg. 2000;48:1-8.
4. Peivandi A, Quinkenstein E, Dahm M, Kasper-König W, Kurocznyski W, Oelert H. Schwere sternale Wundkomplikationen nach kardiochirurgischen Eingriffen. Z Herz-Thorax-Gefäßchir. 2002;16:163-75.

5. Losanoff JE, Jones JW, Richmann BW. Primary closure of median sternotomy: techniques and principles. Cardiovasc Surg. 2002;10:102-10.

6. Lu JC, Grayson AD, Jha P, Srinivasan AK, Fabri BM. Risk factors for sternal wound infection and mid-term survival following coronary artery bypass surgery. Eur J Cardiothorac Surg. 2003;23:943-9.

7. Losanoff JE, Richman BW, Jones JW. Disruption and infection of median sternotomy: a comprehensive review. Eur J Cardiothorac Surg. 2002;21:831-9.

8. Fowler VG, O'Brien SM, Muhlbaier LH, Corey GR, Ferguson TB, Peterson ED. Clinical predictors of major infections after cardiac surgery. Circulation. 2005; 12:358-65.

9. von Hasselbach C. Klinik und Pharmakokinetik von Kollagen-Gentamicin als adjuvante Lokaltherapie knöcherner Infektionen. Unfallchirurg. 1989;92: 459-70.

10. Rutten HJ, Nijhuis PH. Prevention of wound infection in elective colorectal surgery by local application of a gentamicin-containing collagen sponge. Eur J Surg. 1997;578(Suppl):31-5.

11. Bennett-Guerrero E, Pappas TN, Koltun WA, Fleshman JW, Lin M, Garg J, et al. Gentamicin-collagen sponge for infection prophylaxis in colorectal surgery. $N$ Engl J Med. 2010;363:1038-49.

12. Leyh R, Bartels C, Sievers HH. Adjuvant treatment of deep sternal wound infection with collagenous gentamicin. Ann Thorac Surg. 1999;68:1648-51.

13. Eklund A, Valtonen M, Werkkala K. Prophylaxis of sternal wound infections with gentamicin-collagen implant: randomized controlled study in cardiac surgery. J Hosp Infect. 2005;59:108-12.

14. Friberg Ö, Svedjeholm R, Söderquist B, Granfeldt H, Vikerfors T, Källman J. Local gentamicin reduces sternal wound infections after cardiac surgery: a randomized controlled trial. Ann Thorac Surg. 2005;79:153-62.

15. Friberg Ö, Dahlin LG, Källmann J, Kihlströ E, Söderquist B, Svedjeholm R. Collagen-gentamicin implant for prevention of sternal wound infection; longterm follow-up of effectiveness. Interact Cardiovasc Thorac Surg. 2009;9: 454-8.

16. Bennett-Guerrero E, Ferguson TB, Lin M, Garg J, Mark DB, Scavo VA, et al. Effect of an implantable gentamicin-collagen sponge on sternal wound infections following cardiac surgery. JAMA. 2010;304:755-62.

17. Moore R, Lietman P, Smith C. Clinical response to aminoglycoside therapy: importance of the ratio of peak concentration to minimal inhibitory concentration. $J$ Infect Dis. 1987;155:93-9.

18. Penel N, Yazdanpanah Y, Chauvet MP, Clisant S, Giard S, Neu JC, et al. Prevention of surgical site infection after breast cancer surgery by targeted prophylaxis antibiotic in patients at high risk of surgical site infection. J Surg Oncol. 2007;96: 124-9.

19. Holzer B, Grüßner U, Brückner B, Houf M, Kiffner E, Schildberg FW, et al. Efficacy and tolerance of a new gentamicin collagen fleece (Septocoll) after surgical treatment of a pilonidal sinus. Colorectal Dis. 2003;5:222-7.

20. Mangram AJ, Horan TC, Pearson ML, Silver LC, Jarvis WR. Guidelines for prevention of surgical site infection (CDC). Infect Control Hosp Epidemiol. 1999;20:247-78.

21. Graf K, Ott E, Vonberg RP, Kuehn C, Haverich A, Chaberny IF. Economic aspects of deep sternal wound infections. Eur J Cardiothorac Surg. 2010;37: 893-6.

22. Kirklin KB, Briggs JP, Trivette SL, Wilkinson WE, Sexton DJ. The impact of surgical-site infections in the 1990s: attributable mortality, excess length of hospitalization, and extra costs. Infect Control Hosp Epidemiol. 1999;20: 725-30.

23. Coello R, Charlett J, Wilson J, Ward V, Pearson A, Borriello P. Adverse impact of surgical site infections in English hospitals. J Hosp Infect. 2005;60:93-103. 